\title{
A New Approach for Solving Type-2-Fuzzy Transportation Problem
}

\author{
Somnath Maity, Sankar Kumar Roy* \\ Department of Applied Mathematics with Oceanology and Computer Programming \\ Vidyasagar University, Midnapore-721102, West Bengal, India \\ E-mails: somnath1017@gmail.com, *sankroy2006@gmail.com \\ ${ }^{*}$ Corresponding author
}

(Received April 24, 2018; Accepted January 12, 2019)

\begin{abstract}
In this paper, a new approach is introduced to solve transportation problem with type-2-fuzzy variables. In most of the real-life situations, the available data do not happen to be crisp in nature. It gives rise to the fuzzy transportation problem (FTP). This proposed approach concentrates on the problem when the vertical slices of type-2-fuzzy sets (T2FSs) are trapezoidal fuzzy numbers (TFNs). The original problem reduces to three different linear programming problems (LPPs) which are solved using the simplex algorithm. Then the effectiveness of this paper is discussed with numerical example. In conclusion, the significance of the paper and the scope of future study are discussed.
\end{abstract}

Keywords- Transportation problem, Type-2-fuzzy set, Trapezoidal fuzzy number.

\section{Introduction}

The transportation problem (TP) is one of the important linear programming problem which arises in many real-life situations. Hence it has drawn considerable attention in the literature. The core idea of TP is to distribute commodities from certain origins to some destinations in such a way that the total transportation cost gets minimized. The transportation problem has many real-life applications, such as scheduling, production, investment, deciding plant location and inventory control etc. The classical TP has some limitations for designing real-life problem of TP through single objective function where this limitation may be removed by multi objective transportation problem. Many researchers have considered transportation problem with multiple choices and multiple objectives (Mahapatra et al., 2010; Mahapatra et al., 2013; Maity and Roy, 2016; Roy et al., 2017; Roy and Maity, 2017; Roy et al., 2017, Das et al., 2019; Saxena, 2019; Maity et al. 2019). In classical transportation problem, the given data are crisp in nature. However, in real-life situations, most of the cases given data are of imprecise nature. This imprecise nature are measured by fuzzy number in our formulated model.

Zadeh (1975) first introduced the concept of type-2-fuzzy set (T2FS) along with extension principle to extend the basic operations to the set of fuzzy numbers. He also introduced $\alpha$-cut representation which happens to be equivalent with the concept of extension principle. In order to make use of T2FSs in transportation problem, it is needed to introduce meaningful operations for fuzzy sets. Extension principle and $\alpha$-cut representation are the connection between crisp binary operations, such as addition, multiplication and fuzzy binary operations. Xie and Lee (2017) worked on extended type-reduction method for general type-2-fuzzy sets.

Trapezoidal membership functions are commonly used membership functions to represent fuzzy numbers in real-life applications. Triangular membership function can be viewed as a particular type of trapezoidal membership function etc. There are some other types of popular membership 
International Journal of Mathematical, Engineering and Management Sciences

Vol. 4, No. 3, 683-696, 2019

https://dx.doi.org/10.33889/IJMEMS.2019.4.3-054

functions, such as Gaussian membership function, power membership function etc. Triangular, trapezoidal, power, and Gaussian fuzzy numbers are all particular type of LR flat fuzzy numbers. Thus we introduce a new method for modelling and solving FTPs in which all the parameters are T2FSs in nature.

Being a particular type of linear programming problem, fuzzy linear programming approach can be used in solving fuzzy transportation problem. Verdegay (1984) investigated the application of Zimmermann's (2001) fuzzy programming approach and presented the additive fuzzy programming model. Fang et al. (1999) proposed a new method for solving LPPs with fuzzy coefficients in the constraints. Fan et al. (2013) developed a direct transformation algorithm for solving the fuzzy linear programming (FLP) model with uncertainties expressed as fuzzy sets on the left-hand and right-hand sides of the constraints and the objective function. The solution of a fuzzy linear programming should be fuzzy in nature. But, the mentioned techniques come up with crisp solutions which are not expected. Crisp and fuzzy solutions have also been derived for FLP (Gupta and Kumar, 2012; Kumar and Ram, 2018) but no fuzzy solution for the fuzzy transportation problem has been found. As a particular type of LPP, there have been some special types of solution approaches to TP.

Though several researchers have worked on transportation problem under fuzzy environment (Chanas and Kuchta, 1996; Cadenas and Verdegay, 2006; Ebrahimnejad, 2016; Maity and Roy, 2017) but a few of them has worked in type-2-fuzzy environment. In some cases, data cannot be handled with general type-1-fuzzy approaches to transportation problem due to higher order of fuzziness involved with data. As for example, transportation cost may vary in different places, which may depend upon time. Hence type-2-fuzzy set is needed to be introduced for tackling such data. Based on this consideration, a new approach is introduced to handle transportation problem with type-2-fuzzy data. The contributions of this paper are as follows:

- It considers all constraints of the problem to be of type-2-fuzzy nature.

- In the proposed approach, original problem splits into 3 distinct classical LPPs. Combining the three optimal solutions, the optimal solution of the original fuzzy transportation problem is obtained.

- The discussed approach provides a type-2-fuzzy solution. The existing methods give crisp solutions to FTP which should be avoided.

- It assumes a special kind of type-2-fuzzy set whose vertical slices are trapezoidal fuzzy numbers.

- The complexity of computation is reduced significantly using this approach.

The rest of the paper is organized in the following way. In Section 2, some basic definitions are given which will be used throughout the paper. The basic fuzzy operations are discussed in Section 3. The mathematical model of TP is presented in Section 4. In Section 5, the proposed method is discussed with numerical example. Lastly, conclusion is drawn with future scope of study about this paper. 
International Journal of Mathematical, Engineering and Management Sciences

Vol. 4, No. 3, 683-696, 2019

https://dx.doi.org/10.33889/IJMEMS.2019.4.3-054

\section{Basic Definitions}

Here some basic definitions are defined for development of the paper. In this regard, the domains of the fuzzy sets are considered to be the universe of the corresponding fuzzy set, as fuzzy sets are treated as functions in the paper.

Fuzzy Set: A fuzzy subset $F$ of a universe $X$ is a function $F: X \rightarrow[0,1]$.

Crisp Set: A crisp subset $C$ of a universe $X$ is a function $C: X \rightarrow\{0,1\}$ where $C(x)=1$ if $x$ belongs to $A$ and $C(x)=0$ otherwise. We can realize crisp set to be a particular type of fuzzy set.

Trapezoidal Fuzzy Number: Trapezoidal fuzzy number is an ordered 4-tuple $(a, b, c, d)$. It corresponds to a fuzzy subset in the universe of real numbers. Let $A=(a, b, c, d)$. Then $\mu_{A}$ is the fuzzy set corresponding to the fuzzy number $A$, where

$$
\mu_{A}(x)= \begin{cases}\frac{x-a}{b-a} & a \leq x \leq b, \\ 1 & b \leq x \leq c, \\ \frac{d-x}{d-c} & c \leq x \leq d, \\ 0 & \text { otherwise }\end{cases}
$$

The graph of $\mu_{A}$ (Figure 1) takes the form of a trapezium. Hence it is called trapezoidal fuzzy number.

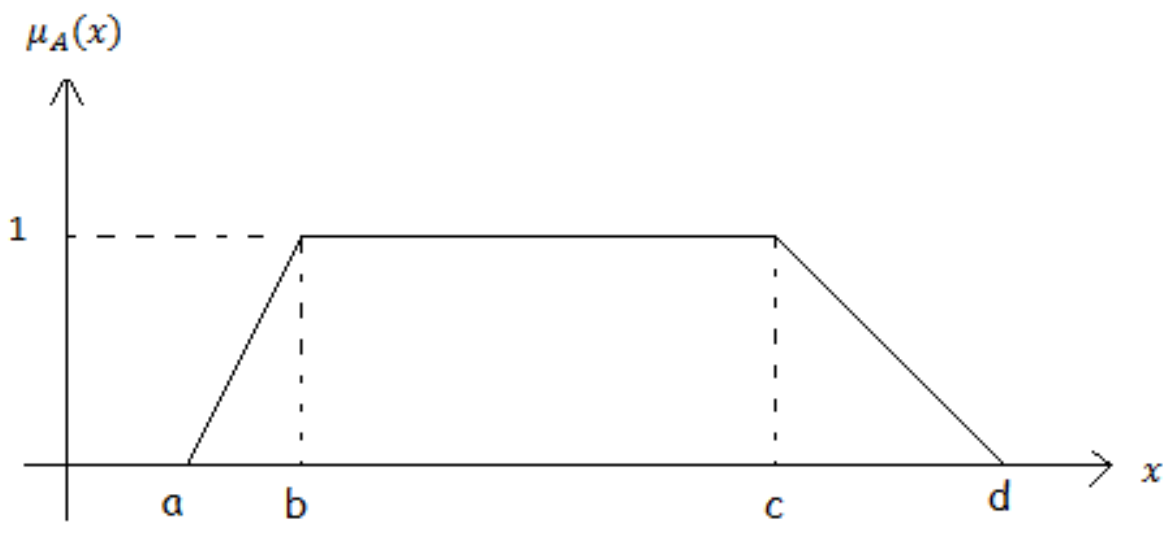

Figure 1. Trapezoidal fuzzy number $(a, b, c, d)$

Extension Principle: Let $X_{1}, X_{2}, \ldots, X_{n}$ be $n$ universes. Let $Y$ be another universe and $f$ be a function $f: X_{1} \times X_{2} \times \ldots \times X_{n} \rightarrow Y$. Let $A_{1}, A_{2}, \ldots, A_{n}$ are fuzzy sets in the universes $X_{1}, X_{2}, \ldots, X_{n}$ respectively. Then we can define a fuzzy set $B_{f}$ in the universe $Y$ using the extension principle. So, $B_{f}$ is given by, 
International Journal of Mathematical, Engineering and Management Sciences

Vol. 4, No. 3, 683-696, 2019

https://dx.doi.org/10.33889/IJMEMS.2019.4.3-054

$$
B_{f}(y)=\sup _{f\left(x_{1}, x_{2}, \ldots, x_{n}\right)=y} \min \left\{A_{1}\left(x_{1}\right), A_{2}\left(x_{2}\right), \ldots, A_{n}\left(x_{n}\right)\right\}
$$

Type-2-Fuzzy Set: Let the universe of all fuzzy sets in $[0,1]$ be $F[0,1]$. A type-2 fuzzy set $T$ in the universe $X$ is a function $T: X \rightarrow F[0,1]$.

$\alpha$-Cut: $\alpha$-cut $(\alpha \in[0,1])$ of a fuzzy set (in the universe $X) A$ is a crisp set $A_{\alpha}$, is given by,

$$
A_{\alpha}(x)= \begin{cases}1 & A(x) \geqslant \alpha \\ 0 & \text { otherwise }\end{cases}
$$

$A$ can be represented in terms of $\alpha$-cut as follows:

$$
A(x)=\sup _{\alpha} \alpha A_{\alpha}(x)
$$

Normalization of Trapezoidal Fuzzy Number: The normalized form of a trapezoidal fuzzy number $(a, b, c, d)$ is given as $\left(0, \frac{b-a}{d-a}, \frac{c-a}{d-a}, 1\right)$. So normalized form, being a fuzzy set itself, can be realized as functional value of a type-2-fuzzy set.

\section{Basic Operations of Fuzzy Sets}

Here we now define some binary operations of fuzzy set in the universe of real numbers using extension principle. Throughout the paper trapezoidal fuzzy number $(a, b, c, d)$ is considered to be positive, i.e., $a \geq 0$.

Addition: Let $A$ and $B$ be two fuzzy sets in the universe of real numbers. Then the addition of two fuzzy sets is defined by, $A \oplus B(z)=\sup _{x+y=z} \min \{A(x), B(y)\}$, where $\bigoplus$ is the fuzzy addition.

Theorem 1: Let $A=\left(a_{1}, a_{2}, a_{3}, a_{4}\right)$ and $B=\left(b_{1}, b_{2}, b_{3}, b_{4}\right)$ be two trapezoidal fuzzy numbers. Then $A \oplus B=\left(a_{1}+b_{1}, a_{2}+b_{2}, a_{3}+b_{3}, a_{4}+b_{4}\right)$.

Proof: Let $\mu_{A}$ and $\mu_{B}$ be the membership functions of $A$ and $B$ respectively.

Then

$\mu_{A}(x)= \begin{cases}\frac{x-a_{1}}{a_{2}-a_{1}} & a_{1} \leq x \leq a_{2} \\ 1 & a_{2} \leq x \leq a_{3} \\ \frac{a_{4}-x}{a_{4}-a_{3}} & a_{3} \leq x \leq a_{4} \\ 0 & \text { otherwise }\end{cases}$

and

$$
\mu_{B}(x)= \begin{cases}\frac{x-b_{1}}{b_{2}-b_{1}} & b_{1} \leq x \leq b_{2} \\ 1 & b_{2} \leq x \leq b_{3} \\ \frac{b_{4}-x}{b_{4}-b_{3}} & b_{3} \leq x \leq b_{4} \\ 0 & \text { otherwise }\end{cases}
$$


International Journal of Mathematical, Engineering and Management Sciences

Vol. 4, No. 3, 683-696, 2019

https://dx.doi.org/10.33889/IJMEMS.2019.4.3-054
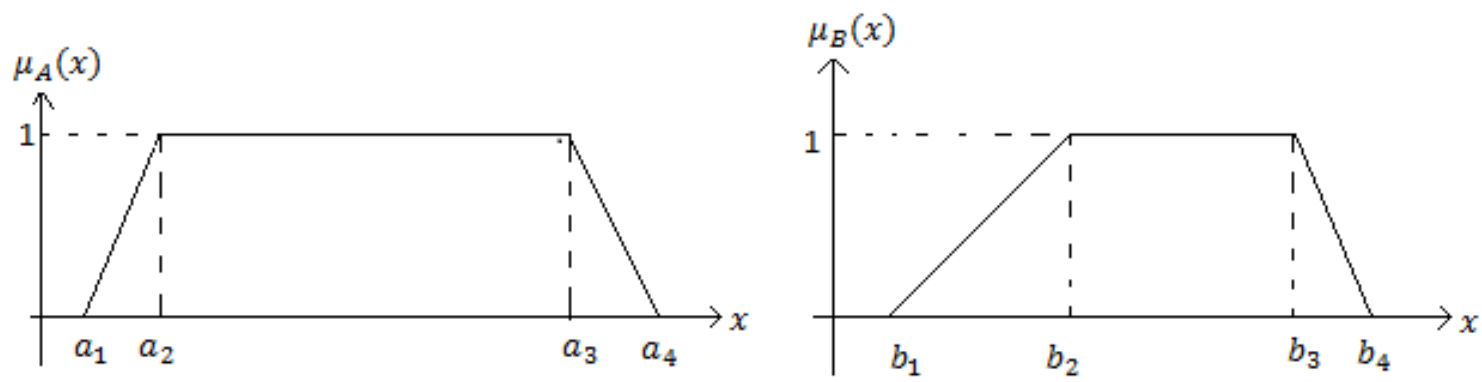

Figure 2. Graphs of trapezoidal fuzzy numbers $A$ and $B$

The graphs of the membership functions $\mu_{A}$ and $\mu_{B}$ are presented in Figure 2.

Let $z \leq a_{1}+b_{1}$. If $x+y=z$ then either $x \leq a_{1}$ or $y \leq b_{1}$ i.e., either $\mu_{A}(x)=0$ or $\mu_{B}(y)=$ 0 . Therefore $\min \left\{\mu_{A}(x), \mu_{B}(y)\right\}=0$. Hence $\sup _{x+y=z} \min \left\{\mu_{A}(x), \mu_{B}(y)\right\}=0$ i.e., $\mu_{A \oplus B}(z)=0$.

Let $a_{1}+b_{1} \leq z \leq a_{2}+b_{2}$. The way trapezoidal fuzzy numbers $A$ and $z-B$ cut each other is shown in Figure 3. If $x+y=z$, then $\sup _{x+y=z} \min \left\{\mu_{A}(x), \mu_{B}(y)\right\}=\sup _{x} \min \left\{\mu_{A}(x), \mu_{B}(z-\right.$ $x)\}$. Let $p$ be the point in $\left(a_{1}, a_{2}\right)$ such that $\sup _{x} \min \left\{\mu_{A}(x), \mu_{B}(z-x)\right\}=\mu_{A}(p)$.
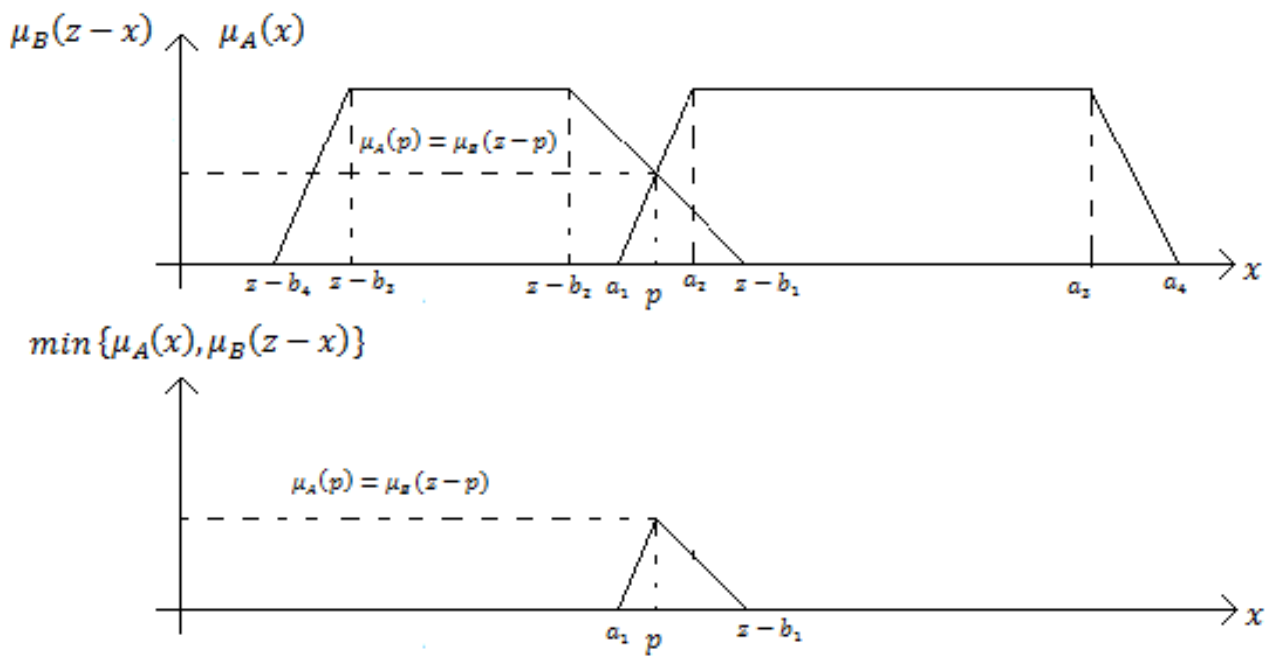

Figure 3. Graphs of the minimum of two trapezoidal fuzzy numbers $A$ and $z-B$ 
International Journal of Mathematical, Engineering and Management Sciences

Vol. 4, No. 3, 683-696, 2019

https://dx.doi.org/10.33889/IJMEMS.2019.4.3-054

Then we have

$\mu_{A}(p)=\mu_{B}(z-p)$

or, $\frac{p-a_{1}}{a_{2}-a_{1}}=\frac{z-p-b_{1}}{b_{2}-b_{1}}=\frac{z-a_{1}-b_{1}}{a_{2}+b_{2}-a_{1}-b_{1}}$.

Therefore $\sup _{x+y=z} \min \left\{\mu_{A}(x), \mu_{B}(y)\right\}=\frac{z-a_{1}-b_{1}}{a_{2}+b_{2}-a_{1}-b_{1}}$, i.e., $\mu_{A \oplus B}(z)=\frac{z-a_{1}-b_{1}}{a_{2}+b_{2}-a_{1}-b_{1}}$.

Let $a_{2}+b_{2} \leq z \leq a_{3}+b_{3}$. Then there exist $a_{2} \leq x \leq a_{3}$ and $b_{2} \leq y \leq b_{3}$ such that $x+y=$ $z$. Hence $\sup _{x+y=z} \min \left\{\mu_{A}(x), \mu_{B}(y)\right\}=1$, i.e., $\mu_{A \oplus B}(z)=1$.

Again let $a_{3}+b_{3} \leq z \leq a_{4}+b_{4}$. If $x+y=z$ then, $\sup _{x+y=z} \min \left\{\mu_{A}(x), \mu_{B}(y)\right\}=$ $\sup _{x} \min \left\{\mu_{A}(x), \mu_{B}(z-x)\right\}$. Let $p$ be the point in $\left(a_{3}, a_{4}\right)$ such that $\sup _{x} \min \left\{\mu_{A}(x), \mu_{B}(z-\right.$ $x)\}=\mu_{A}(p)$, then we have,

$\mu_{A}(p)=\mu_{B}(z-p)$

or, $\frac{a_{4}-p}{a_{4}-a_{3}}=\frac{b_{4}-z+p}{b_{4}-b_{3}}=\frac{a_{4}+b_{4}-z}{a_{4}+b_{4}-a_{3}-b_{3}}$.

Therefore $\sup _{x+y=z} \min \left\{\mu_{A}(x), \mu_{B}(y)\right\}=\frac{a_{4}+b_{4}-z}{a_{4}+b_{4}-a_{3}-b_{3}}$, i.e., $\mu_{A \oplus B}(z)=\frac{a_{4}+b_{4}-z}{a_{4}+b_{4}-a_{3}-b_{3}}$.

Let $z \geq a_{4}+b_{4}$. If $x+y=z$ then either $x \geq a_{4}$ or $y \geq b_{4}$ i.e., either $\mu_{A}(x)=0$ or $\mu_{B}(y)=$ 0 . Therefore $\min \left\{\mu_{A}(x), \mu_{B}(y)\right\}=0$. Hence $\sup _{x+y=z} \min \left\{\mu_{A}(x), \mu_{B}(y)\right\}=0$ i.e., $\mu_{A \oplus B}(z)=0$.

So, the membership function of $\mu_{A \oplus B}$ (Figure 4) is given by,

$$
\mu_{A \oplus B}(x)= \begin{cases}\frac{x-a_{1}-b_{1}}{a_{2}+b_{2}-a_{1}-b_{1}} & a_{1}+b_{1} \leq x \leq a_{2}+b_{2}, \\ \frac{a_{4}+b_{4}-x}{a_{4}+b_{4}-a_{3}-b_{3}} & a_{2}+b_{2} \leq x \leq a_{3}+b_{3}, \\ 0 & \text { otherwise. }\end{cases}
$$

Hence $A \oplus B=\left(a_{1}+b_{1}, a_{2}+b_{2}, a_{3}+b_{3}, a_{4}+b_{4}\right)$.

Multiplication: Similarly, we define multiplication of two fuzzy sets by $A \otimes B(z)=$ $\sup _{x y=z} \min \{A(x), B(y)\}$, where $\otimes$ is the fuzzy multiplication.

Theorem 2: Let $A=\left(a_{1}, a_{2}, a_{3}, a_{4}\right)$ and $B=\left(b_{1}, b_{2}, b_{3}, b_{4}\right)$ be two trapezoidal fuzzy numbers. Then $A \otimes B \simeq\left(a_{1} b_{1}, a_{2} b_{2}, a_{3} b_{3}, a_{4} b_{4}\right)$.

Proof: Let $\mu_{A}$ and $\mu_{B}$ be the membership functions of $A$ and $B$ respectively. The membership functions of $A$ and $B$ are given by equations (1) and (2). 
International Journal of Mathematical, Engineering and Management Sciences

Vol. 4, No. 3, 683-696, 2019

https://dx.doi.org/10.33889/IJMEMS.2019.4.3-054

Let $z \leq a_{1} b_{1}$. If $x y=z$ then either $x \leq a_{1}$ or $y \leq b_{1}$ i.e., either $\mu_{A}(x)=0$ or $\mu_{B}(y)=0$. Therefore $\min \left\{\mu_{A}(x), \mu_{B}(y)\right\}=0 \quad$. Therefore $\sup _{x y=z} \min \left\{\mu_{A}(x), \mu_{B}(y)\right\}=0 \quad$ i.e., $\mu_{A \otimes B}(z)=0$.

Let $a_{1} b_{1} \leq z \leq a_{2} b_{2}$. If $x y=z \quad$ then, $\sup _{x y=z} \min \left\{\mu_{A}(x), \mu_{B}(y)\right\}=\sup _{x} \min \left\{\mu_{A}(x), \mu_{B}(z /\right.$ $x)\}$. Let $p$ be the point in $\left(a_{1}, a_{2}\right)$ such that $\sup _{x} \min \left\{\mu_{A}(x), \mu_{B}(z / x)\right\}=\mu_{A}(p)$, then we have,

$$
\begin{gathered}
\mu_{A}(p)=\mu_{B}(z / p) \\
=\frac{a_{1} b_{2}-b_{1} a_{2}+\sqrt{\left(b_{1} a_{2}-a_{1} b_{2}\right)^{2}+4\left(a_{2}-a_{1}\right)\left(b_{2}-b_{1}\right) z}}{2\left(b_{2}-b_{1}\right)}, \quad[\text { As } p \geq 0] .
\end{gathered}
$$

Therefore $\sup _{x y=z} \min \left\{\mu_{A}(x), \mu_{B}(y)\right\}=\frac{p-a_{1}}{a_{2}-a_{1}}$, i.e., $\mu_{A \otimes B}(z)=\frac{p-a_{1}}{a_{2}-a_{1}}$.

Hence $\mu_{A \otimes} \otimes{ }_{B}(z)=\frac{\sqrt{\left(b_{1} a_{2}-a_{1} b_{2}\right)^{2}+4\left(a_{2}-a_{1}\right)\left(b_{2}-b_{1}\right) z}-a_{1} b_{2}-b_{1} a_{2}-2 a_{1} b_{1}}{2\left(a_{2}-a_{1}\right)\left(b_{2}-b_{1}\right)}$.

Let $a_{2} b_{2} \leq z \leq a_{3} b_{3}$. Then there exist $a_{2} \leq x \leq a_{3}$ and $b_{2} \leq y \leq b_{3}$ such that $x y=z$. Therefore $\sup _{x y=z} \min \left\{\mu_{A}(x), \mu_{B}(y)\right\}=1$, i.e., $\mu_{A \otimes B}(z)=1$.

Let $a_{3} b_{3} \leq z \leq a_{4} b_{4}$. If $x y=z$ then $\sup _{x y=z} \min \left\{\mu_{A}(x), \mu_{B}(y)\right\}=\sup _{x} \min \left\{\mu_{A}(x), \mu_{B}(z /\right.$ $x)\}$. Let $p$ be the point in $\left(a_{3}, a_{4}\right)$ such that $\sup _{x} \min \left\{\mu_{A}(x), \mu_{B}(z / x)\right\}=\mu_{A}(p)$, then

$$
\begin{array}{cc}
\mu_{A}(p)=\mu_{B}(z / p) & \text { or, } \quad p \\
=\frac{b_{4} a_{3}-b_{3} a_{4}+\sqrt{\left(b_{3} a_{4}-b_{4} a_{3}\right)^{2}+4\left(a_{4}-a_{3}\right)\left(b_{4}-b_{3}\right) z}}{2\left(b_{4}-b_{3}\right)},[\text { As } p \geq 0] .
\end{array}
$$

Therefore $\sup _{x y=z} \min \left\{\mu_{A}(x), \mu_{B}(y)\right\}=\frac{a_{4}-p}{a_{4}-a_{3}}$, i.e., $\mu_{A \otimes B}(z)=\frac{a_{4}-p}{a_{4}-a_{3}}$.

Hence $\mu_{A \otimes}{ }_{B}(z)=\frac{2 a_{4} b_{4}-b_{4} a_{3}-b_{3} a_{4}-\sqrt{\left(b_{3} a_{4}-b_{4} a_{3}\right)^{2}+4\left(a_{4}-a_{3}\right)\left(b_{4}-b_{3}\right) z}}{2\left(b_{4}-b_{3}\right)\left(a_{4}-a_{3}\right)}$.

Let $z \geq a_{4} b_{4}$. If $x y=z$ then either $x \geq a_{4}$ or $y \geq b_{4}$ i.e., either $\mu_{A}(x)=0$ or $\mu_{B}(y)=0$. Therefore $\min \left\{\mu_{A}(x), \mu_{B}(y)\right\}=0 \quad$. Therefore $\sup _{x y=z} \min \left\{\mu_{A}(x), \mu_{B}(y)\right\}=0 \quad$ i.e., $\mu_{A \otimes B}(z)=0$.

Hence the membership function of $\mu_{A \otimes}{ }_{B}$ is given by, 
International Journal of Mathematical, Engineering and Management Sciences

Vol. 4, No. 3, 683-696, 2019

https://dx.doi.org/10.33889/IJMEMS.2019.4.3-054

$$
\begin{aligned}
& \mu_{A \otimes B}(x) \\
& = \begin{cases}\frac{\sqrt{\left(b_{1} a_{2}-a_{1} b_{2}\right)^{2}+4\left(a_{2}-a_{1}\right)\left(b_{2}-b_{1}\right) x}-a_{1} b_{2}-b_{1} a_{2}-2 a_{1} b_{1}}{2\left(a_{2}-a_{1}\right)\left(b_{2}-b_{1}\right)} & a_{1} b_{1} \leq x \leq a_{2} b_{2}, \\
1 & a_{2} b_{2} \leq x \leq a_{3} b_{3}, \\
\frac{2 a_{4} b_{4}-b_{4} a_{3}-b_{3} a_{4}-\sqrt{\left(b_{3} a_{4}-b_{4} a_{3}\right)^{2}+4\left(a_{4}-a_{3}\right)\left(b_{4}-b_{3}\right) x}}{2\left(b_{4}-b_{3}\right)\left(a_{4}-a_{3}\right)} & a_{3} b_{3} \leq x \leq a_{4} b_{4}, \\
0 & \text { otherwise. }\end{cases}
\end{aligned}
$$

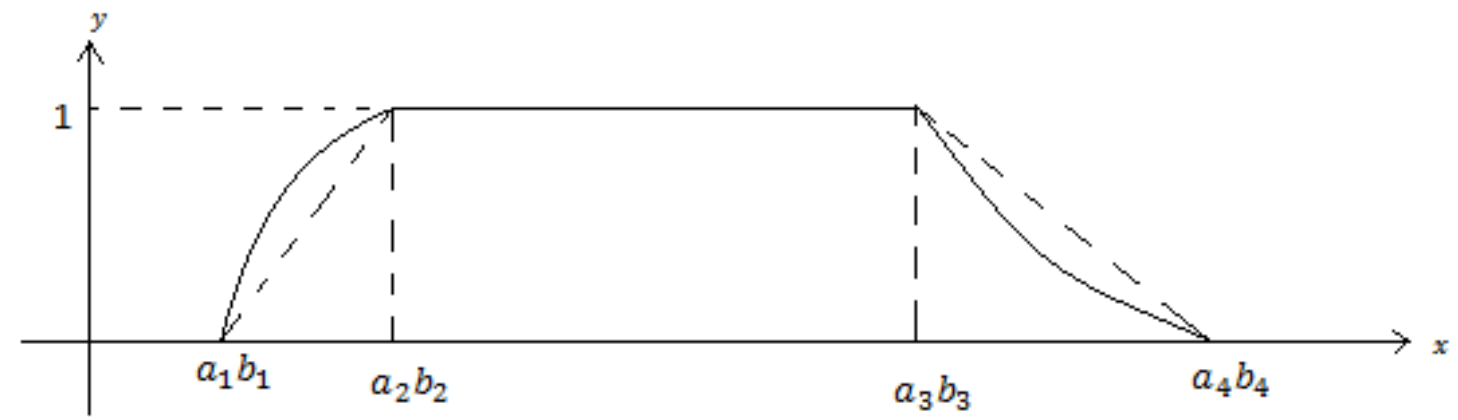

Figure 4. Graph of the fuzzy number $A \otimes B$

The graph of $\mu_{A \otimes B}$ is shown in Figure 4. So the multiplication of two trapezoidal fuzzy numbers is not a trapezoidal fuzzy number. We approximate it by the trapezoidal fuzzy number as $\left(a_{1} b_{1}, a_{2} b_{2}, a_{3} b_{3}, a_{4} b_{4}\right)$.

Though the multiplication of two trapezoidal fuzzy numbers belonging to the set $F[0,1]$ is also an element of $F[0,1]$, but the same is not true for addition. Hence, we modify the definition of fuzzy addition of trapezoidal fuzzy numbers as follows:

The addition of $n$ trapezoidal fuzzy numbers $A_{i}=\left(a_{i 1}, a_{i 2}, a_{i 3}, a_{i 4}\right)$ is given as:

$$
\sum_{i=1}^{n} A_{i}=\left(\frac{1}{n} \sum_{i=1}^{n} a_{i 1}, \frac{1}{n} \sum_{i=1}^{n} a_{i 2}, \frac{1}{n} \sum_{i=1}^{n} a_{i 3}, \frac{1}{n} \sum_{i=1}^{n} a_{i 4}\right) .
$$

Now, some order relations in the set of trapezoidal fuzzy numbers belonging to $F[0,1]$ are needed to be introduced for formulating the proposed model. Our objective is to maximize the length where the trapezoidal fuzzy number takes the value 1 . Hence we define the order as follows:

Let $A=\left(0, a_{1}, a_{2}, 1\right)$ and $B=\left(0, b_{1}, b_{2}, 1\right)$ then $A \geq B$ if $a_{1} \leq b_{1}$ and $a_{2} \geq b_{2}$. 
International Journal of Mathematical, Engineering and Management Sciences

Vol. 4, No. 3, 683-696, 2019

https://dx.doi.org/10.33889/IJMEMS.2019.4.3-054

\section{Model Formulation}

Classical transportation problem was first introduced by Hitchcock (1941) where decision maker is certain about the transportation cost, supply and demand, is formulated as follows:

$$
\begin{aligned}
\text { Minimize } Z= & \sum_{i=1}^{m} \sum_{j=1}^{n} a_{i j} x_{i j} \\
& \text { subject } \text { to } \sum_{j=1}^{n} x_{i j} \leq a_{i}^{1}(i=1,2, \ldots, m), \\
& \sum_{i=1}^{m} x_{i j} \geq a_{j}^{2}(j=1,2, \ldots, n), \\
& x_{i j} \geq 0, \forall i \quad \text { and } j .
\end{aligned}
$$

Where goods are transported from $m$ origins to $n$ destinations, the transportation cost of a unit measure of goods from $i^{\text {th }}$ origin to $j^{\text {th }}$ destination is $c_{i j}$, the supply at $i^{t h}$ origin is $a_{i}^{1}$ and the demand at $j^{\text {th }}$ destination is $a_{j}^{2}$.

In this transportation problem, the decision maker has the privilege of having crisp data for transportation cost, supply and demand. But in real-life situation, the decision maker may not be provided with crisp data. In this model, all the given data and variables are considered to be type2 -fuzzy variables. And the type-2-fuzzy variables are of the form $\left(x, T_{(y, z)}\right)$, where $x, y, z$ are real variables and $T_{(y, z)}$ corresponds to the trapezoidal fuzzy number $(0, y, z, 1)$. Imposing type-2fuzziness in the classical transportation model, type-2-fuzzy transportation model is designed as follows:

\section{Model 1}

$$
\begin{gathered}
\text { Minimize } \tilde{Z}=\left(Z_{1}, T_{\left(z_{2}, z_{3}\right)}\right)=\sum_{i=1}^{m} \sum_{j=1}^{n}\left[\left(a_{i j}, T_{\left(b_{i j}, c_{i j}\right)}\right) \otimes\left(x_{i j}, T_{\left(y_{i j}, z_{i j}\right)}\right)\right] \\
\quad \text { subject to } \sum_{j=1}^{n}\left(x_{i j}, T_{\left(y_{i j}, z_{i j}\right)}\right) \leq\left(a_{i}^{1}, T_{\left(b_{i}^{1}, c_{i}^{1}\right)}\right)(i=1,2, \ldots, m), \\
\sum_{i=1}^{m}\left(x_{i j}, T_{\left(y_{i j}, z_{i j}\right)}\right) \geq\left(a_{j}^{2}, T_{\left(b_{j}^{2}, c_{j}^{2}\right)}\right)(j=1,2, \ldots, n), \\
x_{i j} \geq 0,0 \leq y_{i j} \leq 0.5,0.5 \leq z_{i j} \leq 1, \forall i \quad \text { and } j .
\end{gathered}
$$

The boundary conditions on $y_{i j}$ and $z_{i j}$ are given in order to maintain the consistency in the solutions. If we split the above fuzzy problem in coordinate wise, then we get three mutually different crisp LPPs. Considering the first coordinate $x_{i j}$ we obtain the LPP, is defined in Model 2 as follows:

\section{Model 2}

$$
\begin{aligned}
\text { Minimize } Z_{1}= & \sum_{i=1}^{m} \sum_{j=1}^{n} a_{i j} x_{i j} \\
& \text { subject } \text { to } \sum_{j=1}^{n} x_{i j} \leq a_{i}^{1}(i=1,2, \ldots, m), \\
& \sum_{i=1}^{m} x_{i j} \geq a_{j}^{2}(j=1,2, \ldots, n), \\
& x_{i j} \geq 0, \forall i \quad \text { and } j .
\end{aligned}
$$


International Journal of Mathematical, Engineering and Management Sciences

Vol. 4, No. 3, 683-696, 2019

https://dx.doi.org/10.33889/IJMEMS.2019.4.3-054

It have been already mentioned that our objective would be to maximize $T_{\left(Z_{2}, Z_{3}\right)}$. In order to do that we have to minimize $Z_{2}$ and maximize $Z_{3}$. Considering the second coordinate $y_{i j}$ we obtain the LPP, given by Model 3. $y_{i j}$ corresponds to the minimum point where the trapezoidal fuzzy variable takes the functional value 1 .

\section{Model 3}

Minimize $Z_{2}=\frac{1}{m n} \sum_{i=1}^{m} \sum_{j=1}^{n} b_{i j} y_{i j}$

$$
\begin{aligned}
& \text { subject to } \sum_{j=1}^{n} y_{i j} \geq n b_{i}^{1}(i=1,2, \ldots, m), \\
& \sum_{i=1}^{m} y_{i j} \leq m b_{j}^{2}(j=1,2, \ldots, n) \\
& 0 \leq y_{i j} \leq 0.5, \forall i \quad \text { and } j .
\end{aligned}
$$

Considering the third coordinate $z_{i j}$ we get the LPP, considered in Model 4. $z_{i j}$ corresponds to the maximum point where the trapezoidal fuzzy variable takes the functional value 1 .

\section{Model 4}

$$
\begin{gathered}
\text { Maximize } Z_{3}=\frac{1}{m n} \sum_{i=1}^{m} \sum_{j=1}^{n} c_{i j} z_{i j} \\
\text { subject to } \sum_{j=1}^{n} z_{i j} \leq n c_{i}^{1}(i=1,2, \ldots, m), \\
\sum_{i=1}^{m} z_{i j} \geq m c_{j}^{2}(j=1,2, \ldots, n), \\
0.5 \leq z_{i j} \leq 1, \forall i \quad \text { and } j .
\end{gathered}
$$

Solving three LPPs mentioned in Model 2, Model 3 and Model 4, three optimal solutions $Z_{1}^{*}, Z_{2}^{*}$ and $Z_{3}^{*}$ are obtained respectively. Then the required solution would be $\left(Z_{1}^{*}, T_{\left(Z_{2}^{*}, Z_{3}^{*}\right)}\right)$.

\section{Numerical Example}

Now we illustrate our solution methodology with a suitable numerical example. Let the supply and demand of a product are given in Table 1. Here $O_{1}, O_{2}$ and $O_{3}$ are origins and $D_{1}, D_{2}, D_{3}$ and $D_{4}$ are destinations.

Table 1. Data for transportation cost, supply and demand

\begin{tabular}{|c|c|c|c|c|c|}
\hline Source & $D_{1}$ & $D_{2}$ & $D_{3}$ & $D_{4}$ & Supply \\
\hline$O_{1}$ & $\left(3, T_{(0.15,0.7)}\right)$ & $\left(9, T_{(0.25,0.9)}\right)$ & $\left(22, T_{(0.20,0.85)}\right)$ & $\left(28, T_{(0.35,0.7)}\right)$ & $\left(410, T_{(0.15,0.9)}\right)$ \\
\hline$O_{2}$ & $\left(5, T_{(0.35,0.6)}\right)$ & $\left(7, T_{(0.30,0.8)}\right)$ & $\left(19, T_{(0.1,0.75)}\right)$ & $\left(30, T_{(0.25,0.65)}\right)$ & $\left(400, T_{(0.35,0.8)}\right)$ \\
\hline$O_{3}$ & $\left(15, T_{(0.25,0.9)}\right)$ & $\left(11, T_{(0.30,0.85)}\right)$ & $\left(18, T_{(0.15,0.7)}\right)$ & $\left(32, T_{(0.35,0.6)}\right)$ & $\left(600, T_{(0.25,0.7)}\right)$ \\
\hline Demand & $\left(500, T_{(0.35,0.7)}\right)$ & $\left(300, T_{(0.25,0.8)}\right)$ & $\left(210, T_{(0.25,0.9)}\right)$ & $\left(400, T_{(0.15,0.8)}\right)$ & \\
\hline
\end{tabular}

Utilizing Table 1 in Model 2, we obtain Model 2.1 as follows:

Model 2.1

Minimize $Z_{1}=3 x_{11}+9 x_{12}+22 x_{13}+28 x_{14}+5 x_{21}+7 x_{22}+19 x_{23}$

$$
+30 x_{24}+15 x_{31}+11 x_{32}+18 x_{33}+32 x_{34}
$$


International Journal of Mathematical, Engineering and Management Sciences

Vol. 4, No. 3, 683-696, 2019

https://dx.doi.org/10.33889/IJMEMS.2019.4.3-054

$$
\begin{aligned}
& \text { subject to } x_{11}+x_{12}+x_{13}+x_{14} \leq 410 \\
& x_{21}+x_{22}+x_{23}+x_{24} \leq 400 \\
& x_{31}+x_{32}+x_{33}+x_{34} \leq 600 \\
& x_{11}+x_{21}+x_{31} \geq 500 \\
& x_{12}+x_{22}+x_{32} \geq 300 \\
& x_{13}+x_{23}+x_{33} \geq 210 \\
& x_{14}+x_{24}+x_{34} \geq 400 \\
& 0.5 \leq z_{i j} \leq 1, \forall i \quad \text { and } j .
\end{aligned}
$$

The solution of Model 2.1 is obtained by LINGO 16.0 iterative scheme. The minimum value occurs at the optimal solution $x_{11}=410, x_{24}=10, x_{21}=90, x_{22}=300, x_{31}=390, x_{33}=210, x_{34}=$ 390 and all other $x_{i j}=0$. And the corresponding minimum value of $Z_{1}$ is 20340 .

Using Table 1 in Model 3, we derive Model 3.1 as follows:

\section{Model 3.1}

Minimize $Z_{2}=\frac{1}{12}\left[0.15 y_{11}+0.25 y_{12}+0.20 y_{13}+0.35 y_{14}+0.35 y_{21}+0.30 y_{22}\right.$

$\left.0.35 y_{34}\right]$

$$
+0.1 y_{23}+0.25 y_{24}+0.15 y_{31}+0.30 y_{32}+0.15 y_{33}+
$$

$$
\begin{aligned}
& \text { subject to } y_{11}+y_{12}+y_{13}+y_{14} \geq 0.6, \\
& y_{21}+y_{22}+y_{23}+y_{24} \geq 1.4 \\
& y_{31}+y_{32}+y_{33}+y_{34} \geq 1 \\
& y_{11}+y_{21}+y_{31} \leq 1.05 \\
& y_{12}+y_{22}+y_{32} \leq 0.75 \\
& y_{13}+y_{23}+y_{33} \leq 0.75 \\
& y_{14}+y_{24}+y_{34} \leq 0.45, \\
& 0 \leq y_{i j} \leq 0.5, \forall i \quad \text { and } j .
\end{aligned}
$$

Again the solution of Model 3.1 is calculated using LINGO 16.0 iterative scheme. The minimum value arrives at the optimal solution $y_{11}=0.50, y_{12}=0.10, y_{21}=0.50, y_{22}=0.40, y_{23}=$ $0.50, y_{24}=0.45, y_{31}=0.50, y_{32}=0.25, y_{33}=0.25, y_{34}=0.45$ and all other $y_{i j}=0$. And the corresponding minimum value of $Z_{2}$ is 0.05 .

Taking advantage of Table 1 in Model 4, we obtain Model 4.1 as follows:

\section{Model 4.1}

$$
\begin{aligned}
\text { Maximize } z_{3}=\frac{1}{12}\left[0.7 z_{11}+0.9 z_{12}+\right. & 0.85 z_{13}+0.7 z_{14}+0.6 z_{21}+0.8 z_{22} \\
& +0.75 z_{23}+0.65 z_{24}+0.9 z_{31}+0.85 z_{32}+0.7 z_{33}+ \\
\left.0.6 z_{34}\right] &
\end{aligned}
$$

$$
\begin{aligned}
& \text { subject to } z_{11}+z_{12}+z_{13}+z_{14} \leq 3.6 \\
& z_{21}+z_{22}+z_{23}+z_{24} \leq 3.2 \\
& z_{31}+z_{32}+z_{33}+z_{34} \leq 2.8 \\
& z_{11}+z_{21}+z_{31} \geq 2.1 \\
& z_{12}+z_{22}+z_{32} \geq 2.4
\end{aligned}
$$


International Journal of Mathematical, Engineering and Management Sciences

Vol. 4, No. 3, 683-696, 2019

https://dx.doi.org/10.33889/IJMEMS.2019.4.3-054

$$
\begin{aligned}
& z_{13}+z_{23}+z_{33} \geq 2.7 \\
& z_{14}+z_{24}+z_{34} \geq 2.4, \\
& 0.5 \leq z_{i j} \leq 1, \forall i \text { and } j .
\end{aligned}
$$

The solution of Model 4.1 is obtained by LINGO 16.0 iterative scheme. The maximum value achieves at the point $z_{11}=0.60, z_{12}=1.0, z_{13}=1.0, z_{14}=1.0, z_{21}=0.5, z_{22}=0.80, z_{23}=$ $1.0, z_{24}=0.90, z_{31}=1.0, z_{32}=0.60, z_{33}=0.70, z_{34}=0.50$. And the corresponding maximum value of $Z_{3}$ is 0.65 . So the corresponding optimal solution is $\left(20340, T_{(0.05,0.65)}\right)$.

\section{Conclusions}

In contrast to classical transportation problem, we have considered all the constraints and variables to be type-2-fuzzy variables which has given rise to fuzzy transportation problem. In most of the existing methods, in order to solve the problems, the original problem is transformed into a single crisp problem and which comes up with crisp solution. And in that process we lose significant amount of information which results in deterioration in accuracy of solution. But in our approach, we have considered transportation problem in type-2-fuzzy environment and it comes up with type2 -fuzzy solution. Here the original problem has been converted into three crisp transportation problems and combining the three optimal solutions we have obtained the optimal solution of the original problem.

However, in order to convert into crisp transportation problems, the multiplication of trapezoidal fuzzy number is approximated with a trapezoidal fuzzy number. The approximation is done in a convenient manner to make the computations simplified. In future this flaw can be addressed with some better approximation methods, such as area minimizing method and sup-norm minimizing method etc.

\section{Conflict of Interest}

The authors do not any possible conflicts of interest.

\section{Acknowledgement}

The author, Somnath Maity would like to thank Council of Scientific \&Industrial Research (CSIR) for supporting with financial assistantship to continue this research work under JRF scheme with sanctioned no. 09/599(0068)/2016-EMR-I dated 10/11/2016.

The research of Sankar Kumar Roy is partially supported by the Portuguese Foundation for Science and Technology ("FCT-Fundação para a Ciência e a Tecnologia"), through the CIDMA-Center for Research and Development in Mathematics and Applications, University of Aveiro, Portugal, within project UID/MAT/04106/2019.

\section{References}

Cadenas, J.M., \& Verdegay, J.L. (2006). A primer on fuzzy optimization models and methods. Iranian 
International Journal of Mathematical, Engineering and Management Sciences

Vol. 4, No. 3, 683-696, 2019

https://dx.doi.org/10.33889/IJMEMS.2019.4.3-054

Journal of Fuzzy Systems, 3(1), 1-21.

Chanas, S., \& Kuchta, D. (1996). A concept of the optimal solution of the transportation problem with fuzzy cost coefficients. Fuzzy sets and Systems, 82(3), 299-305.

Das, S.K., Roy, S.K., \& Weber, G.W. (2019). Heuristic approaches for solid transportation-p-facility location problem. Central European Journal of Operations Research, 1-23, https://doi.org/10.1007/s10100-01900610-7.

Ebrahimnejad, A. (2016). New method for solving fuzzy transportation problems with LR flat fuzzy numbers. Information Sciences, 357, 108-124.

Fan, Y.R., Huang, G.H., \& Yang, A.L. (2013). Generalized fuzzy linear programming for decision making under uncertainty: Feasibility of fuzzy solutions and solving approach. Information Sciences, 241, 1227.

Fang, S.C., Hu, C.F., Wang, H.F., \& Wu, S.Y. (1999). Linear programming with fuzzy coefficients in constraints. Computers \& Mathematics with Applications, 37(10), 63-76.

Gupta, A., \& Kumar, A. (2012). A new method for solving linear multi-objective transportation problems with fuzzy parameters. Applied Mathematical Modelling, 36(4), 1421-1430.

Hitchcock, F.L. (1941). The distribution of a product from several sources to numerous localities. Journal of Mathematics and Physics, 20(1-4), 224-230.

Kumar, A., \& Ram, M. (2018). System reliability analysis based on weibull distribution and hesitant fuzzy set. International Journal of Mathematical, Engineering and Management Sciences, 3(4), 513-521.

Maity, G., \& Roy, S.K. (2017). Solving fuzzy transportation problem using multi-choice goal programming. Discrete Mathematics, Algorithms and Applications, 9(6), 1750076.

Maity, G., \& Roy, S.K. (2016). Solving a multi-objective transportation problem with nonlinear cost and multi-choice demand. International Journal of Management Science and Engineering Management, 11(1), 62-70.

Maity, G., Mardanya, D., Roy, S.K., \& Weber, G.W. (2019). A new approach for solving dual-hesitant fuzzy transportation problem with restrictions. Sādhanā, 44(4), 75, DOI: 10.1007/s12046-018-1045-1.

Mahapatra, D.R., Roy, S.K., \& Biswal, M.P. (2013). Multi-choice stochastic transportation problem involving extreme value distribution. Applied Mathematical Modelling, 37(4), 2230-2240.

Mahapatra, D.R., Roy, S.K., \& Biswal, M.P. (2010). Multi-objective stochastic transportation problem involving log-normal. Journal of Physical Sciences, 14, 63-76.

Roy, S.K., Maity, G., Weber, G.W., \& Gök, S.Z.A. (2017). Conic scalarization approach to solve multichoice multi-objective transportation problem with interval goal. Annals of Operations Research, 253(1), 599-620.

Roy, S.K., \& Maity, G. (2017). Minimizing cost and time through single objective function in multi-choice interval valued transportation problem. Journal of Intelligent \& Fuzzy Systems, 32(3), 1697-1709.

Roy, S.K., Maity, G., \& Weber, G.W. (2017). Multi-objective two-stage grey transportation problem using utility function with goals. Central European Journal of Operations Research, 25(2), 417-439.

Saxena, P. (2019). A benchmarking strategy for delhi transport corporation: an application of data envelopment analysis. International Journal of Mathematical, Engineering and Management Sciences, $4(1), 232-244$

Verdegay, J.L. (1984). A dual approach to solve the fuzzy linear programming problem. Fuzzy Sets and Systems, 14(2), 131-141.

Xie, B.K., \& Lee, S.J. (2017). An extended type-reduction method for general type-2 fuzzy sets. IEEE 
International Journal of Mathematical, Engineering and Management Sciences

Vol. 4, No. 3, 683-696, 2019

https://dx.doi.org/10.33889/IJMEMS.2019.4.3-054

Transactions on Fuzzy Systems, 25(3), 715-724.

Zadeh, L.A. (1975). The concept of a linguistic variable and its application to approximate reasoningI. Information Sciences, 8(3), 199-249.

Zimmermann, H.J. (2001). Fuzzy set theory and its applications. $4^{\text {th }}$ Edition, Kluwer Academic Publisher. Dordrecht 